Journal of Nonlinear Optical Physics \& Materials

Vol. 11, No. 1 (2002) 65-74

(C) World Scientific Publishing Company

\title{
PLASMON MODES IN METAL NANOWIRES AND LEFT-HANDED MATERIALS
}

\author{
VIKTOR A. PODOLSKIY \\ E-QUAD Electrical Engineering Department, Princeton University, \\ Princeton, NJ 08544, USA \\ ANDREY K. SARYCHEV and VLADIMIR M. SHALAEV \\ School of Electrical \& Computer Engineering, Purdue University, \\ West Laffayette, IN 47907-1285, USA
}

Received 11 October 2001

\begin{abstract}
The electromagnetic field distribution for thin metal nanowires is found, by using the discrete dipole approximation. The plasmon polariton modes in wires are numerically simulated. These modes are found to be dependent on the incident light wavelength and direction of propagation. The existence of localized plasmon modes and strong local field enhancement in percolation nanowire composites is demonstrated. Novel left-handed materials in the near-infrared and visible are proposed based on nanowire composites.
\end{abstract}

\section{Introduction}

Recently, the problem of EM field distribution for long and thin nanometer-sized needles (nano-wires) has attracted growing interest. ${ }^{1}$ In this paper we are dealing with metal needles, whose diameter is much smaller than the wavelength of incident light, and whose length is of the order of the wavelength. In this limit, we use efficient numerical methods for solving the problem. There are several numerical methods available for finding field distributions for objects of arbitrary shapes. In this paper we use a special modification of the discrete-dipole approximation (DDA), which employs the intersecting effective spheres for finding the field distribution. By using this approach we simulate the field distribution for individual nm-sized metal needles and for a percolation composite formed by such needles. For individual needles, it is found that the surface plasmon polaritons (SPPs) can be excited, resulting in large local fields. For percolation composites formed by the needles, our simulations suggest the existence of localized plasmon modes and strong local field enhancement associated with these modes. The developed theory suggests the possibility of production the left-handed nanowire composite. 


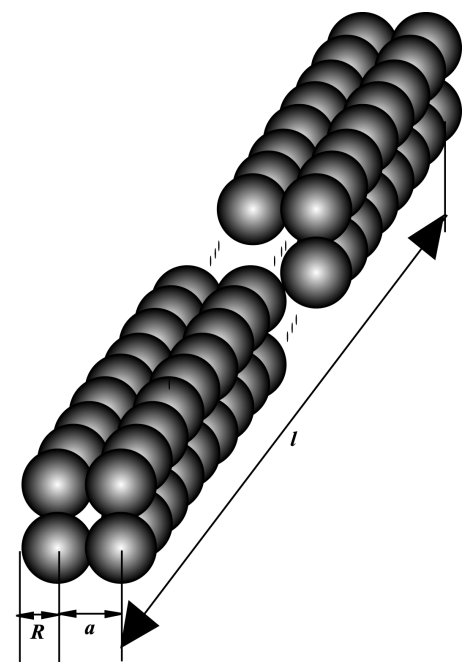

Fig. 1. A long needle modeled by chains of spheres.

The rest of the paper is organized as follows. In the next section we present the coupled-dipole equations (CDEs) used for our calculations and describe a numerical method for finding the EM field distribution. Then, we present and discuss results of our simulations. We further suggest the nanowire composite having negative refractive index in optical and IR spectrum. The last section summarizes main results of the paper.

\section{Coupled-Dipole Equations}

We consider long needles, which can also be referred to as wires. The ratio of the needle length and diameter (the aspect ratio) can vary from 10 to 1000. In our approach, we approximate a long needle by chains of small spheres. In simulations, we use four long chains parallel to each other (see Fig. 1); this, in particular, allows us to take into account the skin effect.

We suppose that the system is illuminated by a monochromatic plane wave $\mathbf{E}_{i n c}(r, t)=\mathbf{E}^{(0)} \exp (i \omega t+i \mathbf{k} \cdot \mathbf{r})$. Below we omit the common factor for all timevarying terms, $\exp (-i \omega t)$. Each spherical particle is then represented by a dipole located at the point $\mathbf{r}_{i}$ (the center of the sphere) with dipole moment $\mathbf{d}_{i}$. This dipole moment is proportional to the local EM field, which is a superposition of the incident EM field and the field "scattered" by all other dipoles of the system. Thus, to find the individual dipole moments one needs to solve the following coupleddipole equations (CDEs)

$$
\mathbf{d}_{i}=\alpha_{0}\left[\mathbf{E}_{i n c}\left(r_{i}\right)+\sum_{j \neq i}^{N} \widehat{G}\left(\mathbf{r}_{i}-\mathbf{r}_{j}\right) \mathbf{d}_{j}\right],
$$


where $\widehat{G}\left(\mathbf{r}_{i}-\mathbf{r}_{j}\right) \mathbf{d}_{j}$ represents the EM field resulting from dipole $j$ at the position of dipole $i$, and $\widehat{G}$ is the regular part of the free-space dyadic Green function defined as

$$
\begin{aligned}
G_{\alpha \beta} & =k^{3}\left[A(k r) \delta_{\alpha \beta}+B(k r) r_{\alpha} r_{\beta} / r^{2}\right], \\
A(x) & =\left[x^{-1}+i x^{-2}-x^{-3}\right] \exp (i x), \\
B(x) & =\left[-x^{-1}-3 i x^{-2}+3 x^{-3}\right] \exp (i x),
\end{aligned}
$$

with $\widehat{G} \mathbf{d}=G_{\alpha \beta} d_{\beta}$. The Greek indices represent the Cartesian components of vectors, and the summation over the repeated indices is implied.

The CDEs approach was first proposed by Purcell and Pennypacker ${ }^{2}$ to find the scattering and absorption of light by odd-shaped dielectric particles. In this approach, the individual dipoles were placed into the cubic lattice within the object, with the lattice period of $a$. The polarizability of an individual dipole was chosen to be equal to the polarizability of a small sphere with radius $R_{m}$. The radius $R_{m}$ is chosen so that the total volume of all spheres is equal to the volume of the object. Note, that the two neighboring particles have to geometrically intersect, in this case, because

$$
a / R_{m}=(4 \pi / 3)^{1 / 3} \approx 1.612<2 .
$$

The polarizability of individual dipoles is given by a well-known ClausiusMossotti relation with the radiative correction introduced by Draine: ${ }^{3}$

$$
\begin{aligned}
\alpha_{L L} & =R_{m}^{3} \frac{\varepsilon-1}{\varepsilon+2}, \\
\alpha_{0} & =\frac{\alpha_{L L}}{1-i\left(2 k^{3} / 3\right) \alpha_{L L}},
\end{aligned}
$$

where $\varepsilon$ is the dielectric constant of the material (the host is assumed to be vacuum), and $\alpha_{L L}$ is the Lorentz-Lorenz polarizability without the radiation correction.

In our case the system is represented by long chains of "intersecting spheres". As shown in Refs. 4 and 5, the "intersection ratio" $a / R_{m}$ for the infinite chain of the spherical particles should slightly differ from the one given by Eq. (3) in order to give the correct depolarization factors

$$
a / R_{m} \approx 1.688
$$

To find the field distribution around a metal needle we are solving the system of linear equations (1) with $\alpha_{0}$ defined in (5)-(4). Numerical methods for solving such equations are described in Ref. 6.

\section{Results of Calculations}

In our simulations the lattice size was chosen to be $15 \mathrm{~nm}$ so that the total crosssection of the needle $(2 \times 2$ particles) is $30 \mathrm{~nm}$ (see Fig. 1). We did two different kinds of simulations. Specifically, we calculated the field distribution over an individual long needle for different angles of incidence of the excitation wave. We also 


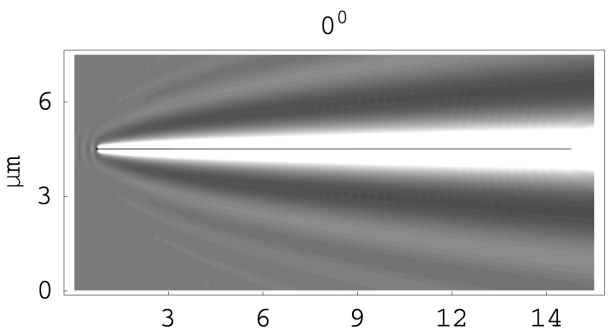

(a)

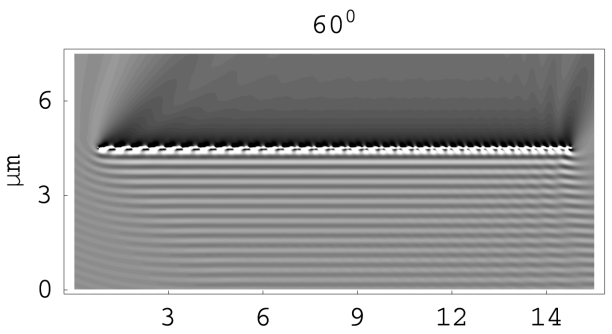

(c)

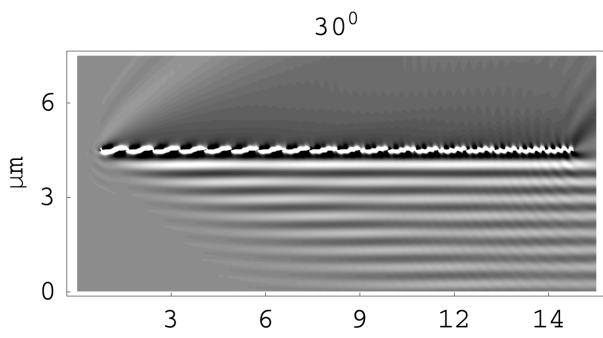

(b)

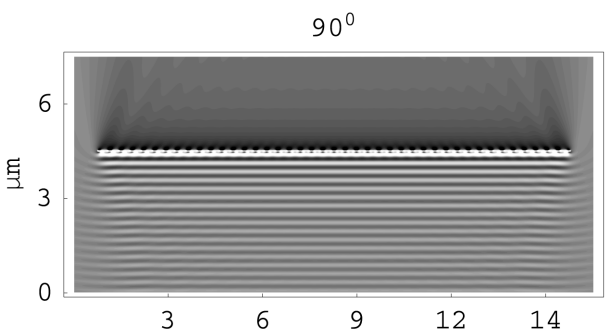

(d)

Fig. 2. EM field distribution for a long needle. The wavelength of incident light is $540 \mathrm{~nm}$. The angle between the wave vector of incident light and the needle is (a) $0^{\circ}$, (b) $30^{\circ}$, (c) $60^{\circ}$ and (d) $90^{\circ}$.

calculated the field distribution over a percolation composite made from randomly distributed needles. In all our simulations the wavelength of incident light is $540 \mathrm{~nm}$.

We first address the question of the EM field distribution for a single metal needle. For this kind of simulations we used a very long needle, with the length $15 \mu \mathrm{m}$. In all pictures obtained we can clearly see the interference pattern between the incident wave and SPP wave in the needle (see Fig. 2). This pattern strongly depends on the angle between the needle and the wave vector of the incident wave (note that the needle and the wave vector of the incident light always lie in the same plane). In Fig. 2(a) the wave vector of the incident light is parallel to the needle. In Figs. 2(b) and 2(c) the angle between them is 30 and 60 degrees, respectively. Figure 2(d) shows the field distribution for the case when the wave vector of the incident light is perpendicular to the needle (in this case the electric field is parallel to the needle).

Our simulations suggest the existence of plasmon resonances in the metal needle. To check this assumption we varied the needle length, keeping the wavelength of the incident light and the angle of incidence both fixed. The angle of incidence was chosen to be 30 degrees. To save the computational time we used in these calculations "short" needles, with the length close to the wavelength (Figs. 3(a), $3(\mathrm{~b})$ and $3(\mathrm{c}))$. Note that the length and diameter ratio, in this case, is close to 15 ; so it is still much larger than 1 . Our results show the existence of the 

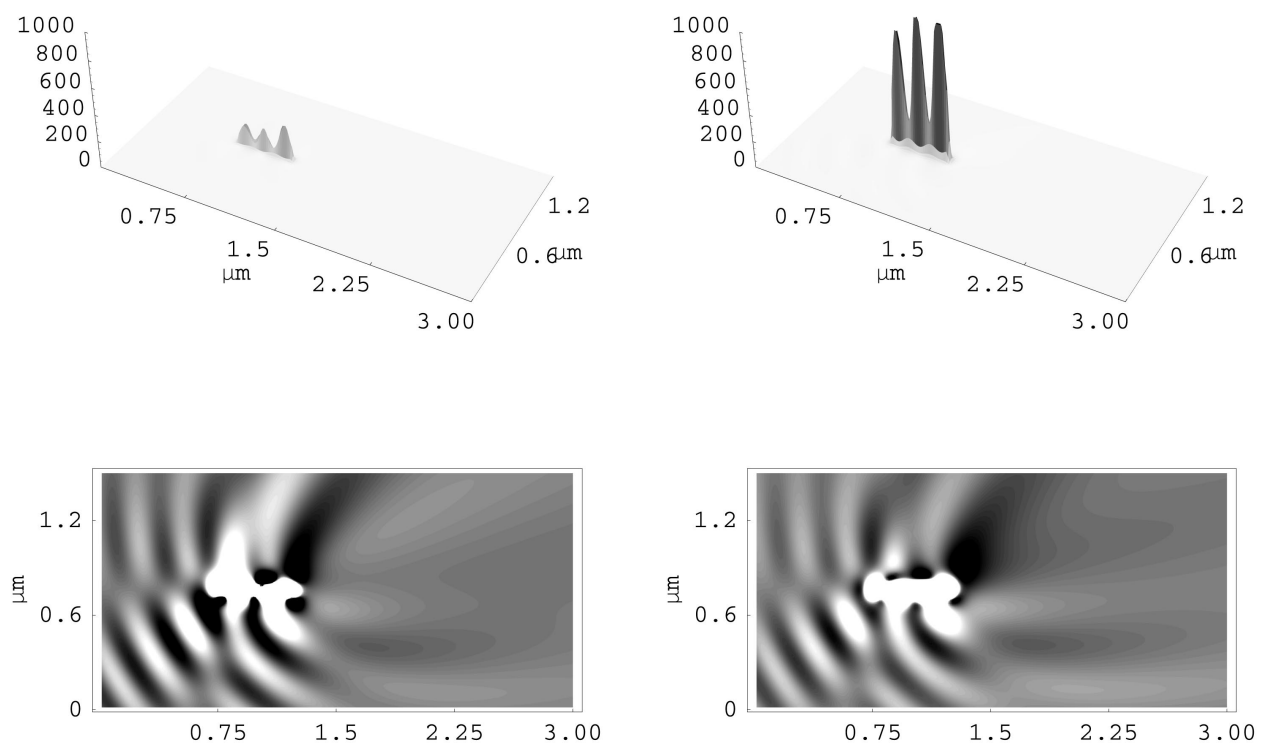

(a)

(b)

Needle Length $=495 \mathrm{~nm}$
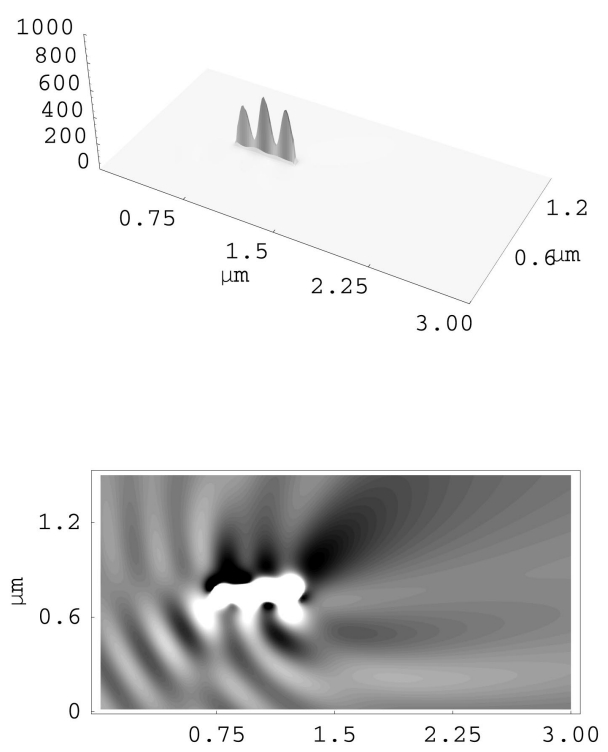

(c)

Fig. 3. Plasmon resonance in a "short" needle. The wavelength of incident light is $540 \mathrm{~nm}$, the angle of incidence is $30^{\circ}$. The needle length is (a) $465 \mathrm{~nm}$, (b) $480 \mathrm{~nm}$ and (c) $495 \mathrm{~nm}$. 
resonance when the needle length is an integer of the half of the plasmon wavelength. Also, we obtained strong dependence of the resonant needle length on the angle of incidence. The resonances are very sharp with the peak intensity enhancement on the order of $10^{3}$. We checked our results for different angles of incidence and found similar resonant behavior. We also conducted simulations, with the length of the needle being constant, whereas the wavelength of the incident light was varying; the obtained results show similar resonant behavior.

The next part of our work was to obtain the EM field distribution over randomly distributed metal needles on the plane. The concentration of needles was chosen to be equal to the percolation (conductivity) threshold. The existence of localized plasmon modes for metal-dielectric percolation films (also referred to as semicontinuous metal films) was recently predicted by Sarychev and Shalaev and experimentally verified in experiments performed by a French team (see Refs. 7 and 8 and references therein). In semicontinuous metal films local fields are shown to be extremely enhanced in small nm-sized areas. We anticipate similar kind of behavior in a percolation 2D system composed of needles. Percolation threshold for the "needle composite" is known to be equal to $b / l$, where $b$ is the needle diameter and $l$ is the needle length (see Ref. 9 and references therein).

In our numerical simulations we considered the "needle composite" exactly at the percolation threshold. The length of individual needles in the composite was chosen to be equal to the resonant needle length for the case when the electric field in the incident wave is parallel to the needle. Our results are presented in Fig. 4. In Fig. 4(a), we show the system of needles used for simulations. The spatial EM field distribution is presented in Fig. 4(b). In Fig. 4(c), we show results of simulations for the near-field scanning optical microscope (NSOM) image. As seen in the figures, the simulation results suggest the existence of localized plasmon modes in the system, with the peak intensity enhancement on the order of $10^{3}$ in the near field.

\section{Nanowire Composites with Negative Refractive Index}

Shultz and Smith, following and further developing ideas of Pendry have recently demonstrated a new material, having in the $\mathrm{GHz}$ spectral range permittivity, $\varepsilon$, and magnetic permeability, $\mu$, both negative. ${ }^{11}$ Such material should also have negative refractive index, $n=-\sqrt{\varepsilon \mu}$. As first predicted by Veselago, the electromagnetic plane wave in such media propagates in the direction opposite to the flow of energy. Such materials are often called "left-handed" since the three vectors $E, H$ and $k$ inside them form a left-handed system. These materials are anticipated to have a number of very unusual properties, which include inverse Doppler shift, inverse Snell effect, and reversed Cerenkov radiation. A plane slab of such material can focus a light beam. ${ }^{11}$

In this section, we describe a material comprising pairs of nanowires parallel to each other. We show that such material can have a negative refractive index in the 


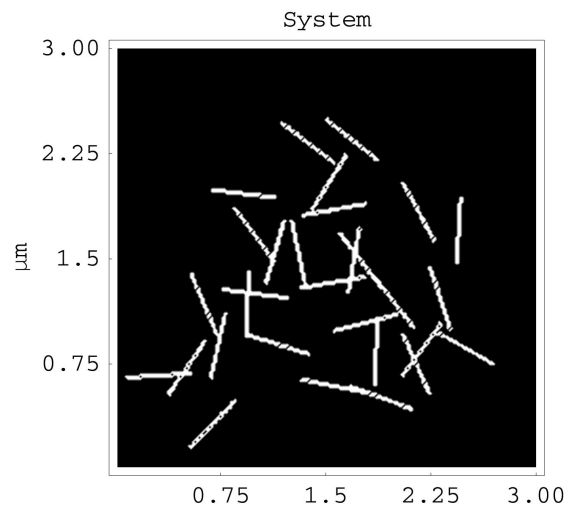

(a)

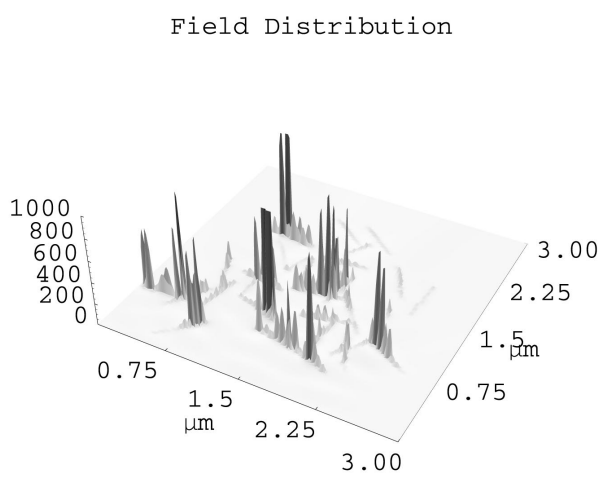

(b)

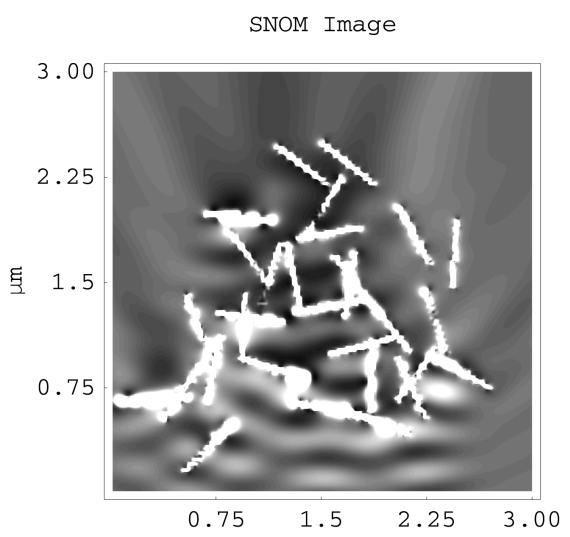

(c)

Fig. 4. (a) Percolation composite formed by metal needles; (b) the EM field distribution for the composite and (c) NSOM image simulation.

near IR and the visible spectral ranges. Consider a thin layer of material, composed from pairs of nanowires parrallel to each other. The length of individual nanowires is $2 b_{1}$, their diameter is $2 b_{2}$, and the distance between the nanowires in the pair is $d$. The needles are assumed to be embedded into host with dielectric constant $\varepsilon_{d}$. We consider the case of closely placed long nanowires so that $b_{2} \ll d \ll b_{1}$. The incident wave propagates normal to the composite surface so that the electric field is parallel to the nanowires, while the magnetic field is perpendicular to the nanowire pairs (see Fig. 5). We consider the macroscopic optical characteristics of such material, namely, the effective dielectric constant $\varepsilon$ and effective magnetic permeability $\mu$. To take into account a skin-effect we introduce the function ${ }^{12} f(\Delta)=\frac{(1-i)}{\Delta} \frac{J_{1}[(1+i) \Delta]}{J_{0}[(1+i) \Delta]}$, where parameter $\Delta=b_{2} \sqrt{2 \pi \sigma_{m} \omega} / c \gg 1$ represents the ratio of nanowire radius and the skin depth $\left(\sigma_{m}\right.$ is bulk metal conductivity). 

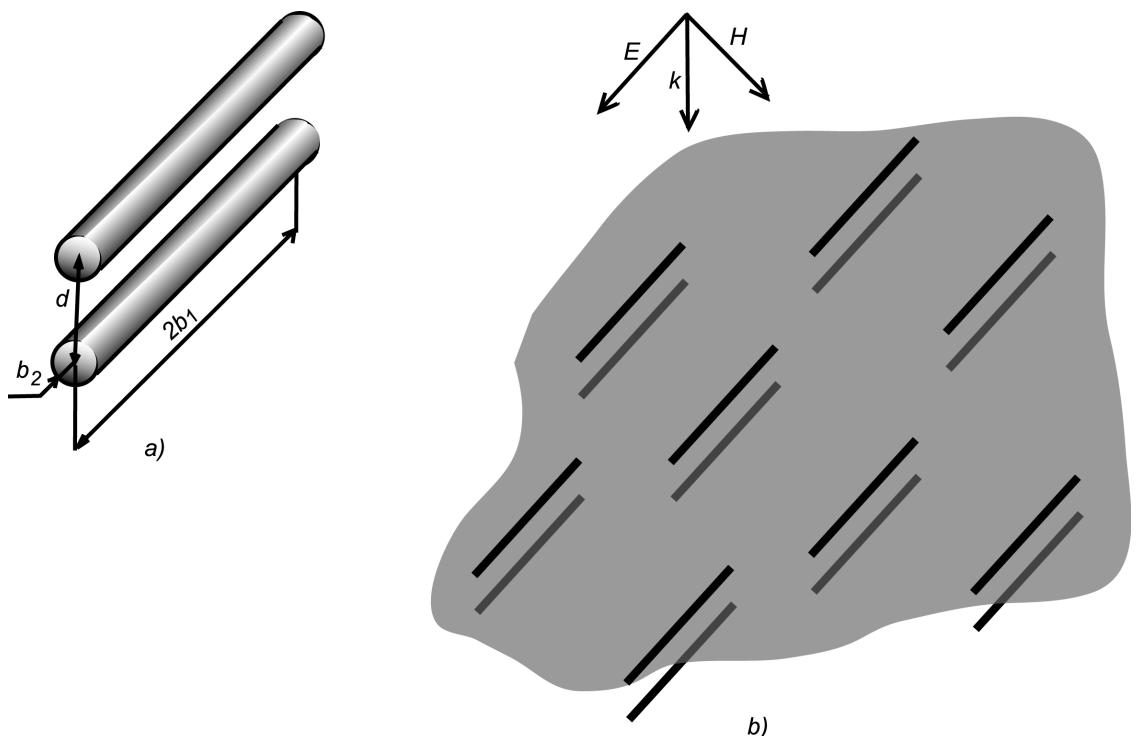

Fig. 5. (a) System of two parallel nanowires and (b) a composite comprising such pairs of parallel nanowires.

To find the magnetic permeability of the composite we first find the magnetic moment $m_{H}$ of the individual two-needle system. We approximate such pair by two parallel infinite wires so that the telegraph equation can be applied. ${ }^{9}$ The time-varying magnetic field excites the currents in the nanowires in the pairs, with the displacement currents between the wires "closing" the electric circuit. Thus, the currents induced by the magnetic field move in opposite directions in the wires in the pair. Using the telegraph equation we arrive at the following expression for the magnetic moment of the pair (for details, see Refs. 9 and 10).

$$
m_{H}=2 H b_{1}^{3} C_{2}(k d)^{2} \frac{\tan \left(g b_{1}\right)-g b_{1}}{\left(g b_{1}\right)^{3}}
$$

where $C_{2}=\frac{\varepsilon_{d}}{4 \ln \left(d / b_{2}\right)}$ is the system's capacity per unit length, and parameter $g$ is given by $g=k \sqrt{\varepsilon_{d}+i \frac{\varepsilon_{d}}{2 \Delta^{2} f(\Delta) \ln \left(d / b_{2}\right)}}$.

The incident electric field is parallel to the wires, so it excites equal currents in the two wires in the pair, which can be considered as independent. The total dipole moment for the two nanowires in the pair is given by (see Ref. 9)

$$
d_{E}=\frac{2}{3} b_{1} b_{2}^{2} f(\Delta) E \varepsilon_{m} \frac{1}{1+f(\Delta) \varepsilon_{m}\left(b_{2} / b_{1}\right)^{2} \ln \left(1+\varepsilon_{d} b_{1} / b_{2}\right) \cos \Omega},
$$

where the dimensionless frequency is given by $\Omega^{2}=\left(b_{1} k\right)^{2} \frac{\ln \left(b_{1} / b_{2}\right)+i \sqrt{\varepsilon_{d}} k b_{1}}{\ln \left(1+\varepsilon_{d} b_{1} / b_{2}\right)}$. 


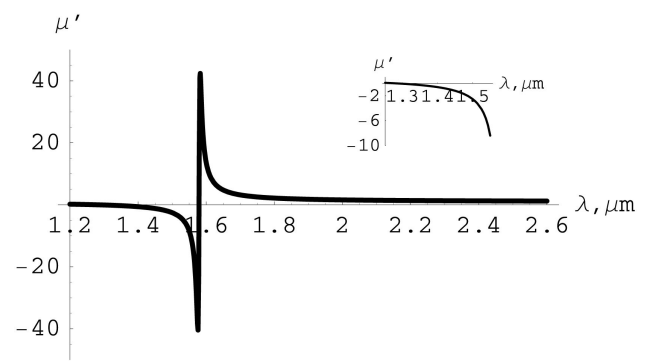

(a)

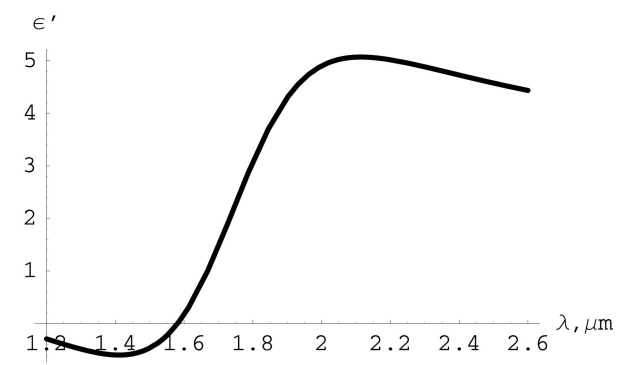

(b)

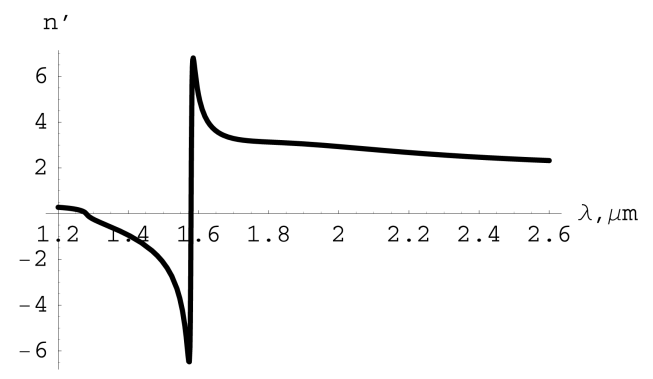

(c)

Fig. 6. (a) The real parts of the effective magnetic permeability, (b) dielectric permittivity, (c) and refractive index for the composite shown in Fig. 5(b). Surface metal concentration $p=0.1$; other parameters are as follows $b_{1}=350 \mathrm{~nm}, b_{2}=5 \mathrm{~nm}$, and $d=150 \mathrm{~nm}$.

Using the magnetic and dipole moments above, we can find the effective dielectric constant and magnetic permeability for the 2D nanoneedle composite film (see also Ref. 13)

$$
\begin{aligned}
& \varepsilon=1+\frac{4 p}{b_{1} b_{2} d} \frac{d_{E}}{E}, \\
& \mu=1+\frac{4 p}{b_{1} b_{2} d} \frac{m_{H}}{H},
\end{aligned}
$$

where $p$ is surface metal concentration.

The macroscopic parameters of the composite, which are calculated using the formulas above, are shown in Fig. 6. As seen in the figure, both permittivity and magnetic permeability have the resonant structure. Note that the resonance position (and therefore the spectal range where the material refractive index is negative) is determined by parameters $b_{1}, b_{2}$ and $d$. In Fig. 6 we illustrate the left-handed material in a vicinity to the telecommunication wavelength $1.5 \mu \mathrm{m}$. By varying the parameters $b_{1}, b_{2}$ and $d$ the negative-refraction spectral range can be moved to the visible part of the spectrum. 


\section{Conclusions}

The EM field distribution over thin metal needles (wires) was numerically simulated. Results show the existence of propagating plasmon polariton modes in the needles. These modes are strongly dependent on the incident wave characteristics, such as wavelength and angle of incidence. The existence of plasmon resonance and its strong dependence on the incident field characteristics is also shown. Strong localfield enhancement is found in the resonance.

The field distribution over a percolation composite formed by metal needles is also studied. The results obtained suggest the existence of localized plasmon modes with the local intensity enhancement factors on the order of $10^{3}$.

We also proposed and studied novel nanowire-based composites with a negative refractive index in the near-infrared and the visible.

\section{Acknowledgments}

This work was supported in part by NSF grant DMR-0121814, by NASA grant NCC-1-01049, and by ARO grant DAAD 19-01-1-0682. Authors are also grateful to Martin Moskovits for suggesting the problem and useful discussions.

\section{References}

1. S. D. M. Brown, P. Corio, A. Marucci, M. A. Pimenta, M. S. Dresselhaus and G. Dresselhaus, Phys. Rev. B61, 7734 (2000); M. S. Dresselhaus, G. Dresselhaus and M. A. Pimenta, European Phys. J. D9, 69 (1999); K. B. Shelimov and M. Moskovits, Chem. Mat. 12, 250 (2000); J. Li, C. Papadopoulos, J. M. Xu and M. Moskovits, Appl. Phys. Lett. 75, 367 (1999); and references therein.

2. E. M. Purcell and C. R. Pennypacher, Astrophys. J. 405, 705 (1973).

3. B. T. Draine, Astrophys. J. 333, 848 (1988).

4. V. A. Markel, J. Opt. Soc. Am. B12, 1783 (1995).

5. V. A. Markel, J. Mod. Opt. 39, 853 (1992).

6. V. A. Markel and V. M. Shalaev, "Computational approaches in optics of fractal clusters", in Computational Studies of New Materials, eds. D. A. Jelski and T. F. George (World Scientific, Singapore, 1999).

7. V. M. Shalaev, Nonlinear Optics of Random Media: Fractal Composites and MetalDielectric Films (Springer Verlag, STMP-158, Berlin, Heidelberg, 2000).

8. S. Ducourtieux, V. A. Podolskiy, S. Grésillon, B. Berini, P. Gadenne, A. C. Boccara, J. C. Rivoal, W. A. Bragg, K. Banerjee, V. P. Safonov, V. P. Drachev, Z. C. Ying, A. K. Sarychev and V. Vladimir M. Shalaev, Phys. Rev. B64, 165403 (2001).

9. A. N. Lagarkov and A. K. Sarychev, Phys. Rev. B53, 10 (1996).

10. D. P. Makhovskiy, L. V. Panina, D. J. Mapps and A. K. Sarychev, Phys. Rev. B64, 134205 (2001).

11. D. R. Smith, W. J. Padilla, D. C. Vier, S. C. Nemat-Nasser and S. Shultz, Phys. Rev. Lett. 84, 4184 (2000); D. R. Smith and N. Kroll, Phys. Rev. Lett. 85, 2933 (2002); J. B. Pendry, Phys. Rev. Lett. 85, 3966 (2000).

12. L. D. Landau and E. M. Lifshitz, Electrodynamics of Continuous Media, 2nd ed. (Pergamon, Oxford, 1984).

13. A. P. Vinogradov, Physica A241, 216 (1997). 